\title{
Clinical predictors of microvascular obstruction by delayed enhanced CMR in STEMI patients
}

\author{
Maria M Izquierdo ${ }^{1 *}$, José T Ortiz-Pérez ${ }^{2}$, Lara Bakhos ${ }^{1}$, Alejandro Aquino ${ }^{1}$, Ander Regueiro ${ }^{2}$, Teresa De Caralt ${ }^{2}$, \\ Xavier Bosch², Daniel C Lee', Edwuin Wu', Rosario Jesus Perea², Susana Prat ${ }^{2}$ \\ From 2011 SCMR/Euro CMR Joint Scientific Sessions \\ Nice, France. 3-6 February 2011
}

\section{Background}

The presence of microvascular obstruction (MO) on cardiac magnetic resonance (CMR) imaging is associated with adverse remodeling and poor prognosis after STEMI. Identifying which patients that may develop MO prior to undergoing acute mechanical reperfusion maybe important in managing patients for more direct interventions. We sought to evaluate clinical predictors of CMR identified MO.

\section{Methods}

We included 255 patients with their first STEMI reperfused with primary percutaneous intervention. A standard DE-CMR was performed acutely at a mean of $3.9 \pm 2.0$ days after admission. Clinical risk factors, time to reperfusion as well as angiographic variables were prospectively collected. The angiographic area at risk and the infarct size as a \% of the left ventricle (LV) were computed. The number of segments with MO, defined as an area of hypoenhancement surrounded by delayed enhancement on DE-CMR, were summed to calculate MO extent.

\section{Results}

MO was present in $44 \%$ of the cases. Patients with MO had $3.1 \pm 1.8$ segments with MO. Different variables were analyzed but only male gender, diabetes, infarct location anterior, initial TIMI 0/1 flow and absent collaterals (Rentrop's grades $0 / 1$ ) were associated with a greater $\mathrm{MO}$ extent. In addition, greater number of $\mathrm{MO}$ segments significantly correlated with a larger angiographic area at risk $(\mathrm{P}=0.005)$ and infarct size $(\mathrm{P}<0.001)$ and borderline with time to reperfusion $(\mathrm{P}=0.06)$.

'Department of Medicine, Division of Cardiology, Northwestern University,

Chicago, IL, USA

Full list of author information is available at the end of the article
Table 1 Number of segments with MO \pm standard deviation

\begin{tabular}{llll}
\hline & Yes & No & Pvalue \\
\hline Male gender & $1.5 \pm 2.0$ & $0.7 \pm 1.5$ & $<0.01$ \\
Diabetes & $1.9 \pm 1.9$ & $1.2 \pm 1.9$ & $<0.01$ \\
Anterior location & $1.6 \pm 2.2$ & $0.8 \pm 1.3$ & $<0.01$ \\
Initial TIMI flow 0/1 & $1.5 \pm 2.0$ & $0.8 \pm 1.5$ & 0.03 \\
Absent collaterals & $1.5 \pm 2.1$ & $0.8 \pm 1.4$ & 0.02 \\
\hline
\end{tabular}

Important nonsignificant parameters include hypertension, tobacco use, hypercholesterolemia, family history of CAD or final TIMI epicardial flow. By multivariate regression analysis, the area at risk, male gender, diabetes, absence of collaterals and initial poor TIMI flow remained as the only significant independent predictors of MO extent.

\section{Conclusions}

The prevalence of MO is often seen in STEMI. Larger MO sizes are seen in diabetics and males, as well as in patients with large areas at risks and poor residual flow prior to mechanical revascularization.

\section{Author details}

'Department of Medicine, Division of Cardiology, Northwestern University, Chicago, IL, USA. ${ }^{2}$ Thorax Institute, Hospital Clinic i Provincial , Barcelona University, Barcelona, Spain.

Published: 2 February 2011

doi:10.1186/1532-429X-13-S1-P115

Cite this article as: Izquierdo et al: Clinical predictors of microvascular obstruction by delayed enhanced CMR in STEMI patients. Journal of Cardiovascular Magnetic Resonance 2011 13(Suppl 1):P115.

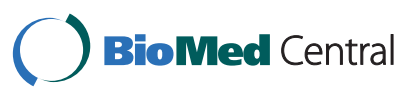

(C) 2011 Izquierdo et al; licensee BioMed Central Ltd. This is an open access article distributed under the terms of the Creative Commons Attribution License (http://creativecommons.org/licenses/by/2.0), which permits unrestricted use, distribution, and reproduction in any medium, provided the original work is properly cited. 